Diego Orlando

\title{
Absorsor Pendular para Controle de Vibrações de Torres
}

Esbeltas

Dissertação de Mestrado

Dissertação apresentada como requisito parcial para obtenção do título de Mestre pelo Programa de PósGraduação em Engenharia Civil da PUC-Rio. Área de Concentração: Estruturas.

Orientador: Paulo Batista Gonçalves

Rio de Janeiro, março de 2006 
Diego Orlando

\title{
Absorsor Pendular para Controle de Vibrações de Torres \\ Esbeltas
}

\begin{abstract}
Dissertação apresentada como requisito parcial para obtenção do título de Mestre pelo Programa de PósGraduação em Engenharia Civil da PUC-Rio. Aprovada pela Comissão Examinadora abaixo assinada.
\end{abstract}

Prof. Paulo Batista Gonçalves

Presidente/Orientador

Departamento de Engenharia Civil - PUC-Rio

Prof. Carlos Magluta

Universidade Federal do Rio de Janeiro - COPPE-UFRJ

Prof. João Luis Pascal Roehl

Departamento de Engenharia Civil - PUC-Rio

Prof. Raul Rosas e Silva

Departamento de Engenharia Civil - PUC-Rio

Prof. José Eugênio Leal

Coordenador(a) Setorial do Centro Técnico Científico - PUC-Rio

Rio de Janeiro, 03 de março de 2006 
Todos os direitos reservados. É proibida a reprodução total ou parcial do trabalho sem autorização da universidade, do autor e do orientador.

Diego Orlando

Graduou-se em Engenharia Civil pela Universidade de Passo Fundo (UPF), em janeiro de 2004. Participou de projetos de iniciação científica no Laboratório de Ensaios em Sistemas Estruturais (LESE-UPF). Ingressou no curso de mestrado em Engenharia Civil da PUC-Rio em março de 2004, atuando na área de Dinâmica Estrutural e Controle de Vibrações.

Ficha Catalográfica

Orlando, Diego

Absorsor pendular para controle de vibrações de torres esbeltas / Diego Orlando ; orientador: Paulo Batista Gonçalves. - Rio de Janeiro : PUC, Departamento de Engenharia Civil, 2006.

168 f. : il. ; $30 \mathrm{~cm}$

Dissertação (mestrado) - Pontifícia Universidade Católica do Rio de Janeiro, Departamento de Engenharia Civil.

Inclui referências bibliográficas.

1. Engenharia civil - Teses. 2. Torres esbeltas. 3. Absorsor dinâmico de vibrações. 4. Absorsor pendular. 5. Controle de vibrações. 6. Oscilações não-lineares. 7. Estabilidade dinâmica. I. Gonçalves, Paulo Batista. II. Pontifícia Universidade Católica do Rio de Janeiro. Departamento de Engenharia Civil. III. Título. 
Dedico este trabalho como minha mais saudosa homenagem aos meus pais, Wilson Orlando e Melânia Maria Orlando, por todo amor, carinho e auxílio no decorrer da minha vida. Para meu irmão Thiago Orlando, pela amizade e por todas as oportunidades de brincadeira e descontração. 


\section{Agradecimentos}

Agradeço a vida, e àqueles que passam fazendo-a valer a pena.

Ao Professor Paulo Batista Gonçalves pelas conversas, pelo auxílio constante na realização deste trabalho, pela paciência e por sua amizade.

Aos professores do Departamento de Engenharia Civil da PUC-Rio pelos ensinamentos transmitidos.

Aos professores que participaram da Comissão examinadora.

A meus familiares que sempre acreditaram em mim, em especial a minhas avós e a meus avôs (Fidelis Orlando e Bonifácio Popiolek, in memoriam). A minha "Tia Ninha”, que ainda está viva em minha memória.

Aos meus amigos de uma vida inteira, em especial Eduardo de Mattos, Erblai de Mattos Junior, Cleiton Batista Silverio, Henrique Marek, André Guimarães, Eduardo Zimmer, Maikel Orlando, Célio França, Taiana França, Denise Marek, Carla Dall’Agnol, Osmar Cervieri e Jaime Giolo.

Aos Professores, Engenheiros e amigos Zacarias Chamberlain e Gilnei Artur Drehmer pelo constante apoio e incentivo.

Aos colegas e amigos que colaboraram nessa Dissertação em especial Frederico Martins, André Muller, Eduardo Pasquetti, Walter Menezes e Igor Otiniano.

Aos grandes amigos Julio e Gisele Holtz, Patrícia Cunha, Fernando Ramires e Alexandre Del Savio obrigado pelo incentivo e apoio.

Aos colegas, companheiros e amigos de festa e descontração Adriano, Thiago Pecin, Ygor, Christiano, Tiago Proto e Adenilson.

Aos antigos companheiros de republica Tinho, Zé, Fred, Pasquetti e Magnus, por terem me aturado tanto tempo e aos novos colegas de apartamento Thiago, Erblai 
e Luis Gustavo.

A Cnpq e a Capes pelo apoio financeiro, sem os quais este trabalho não poderia ser realizado.

A PUC-Rio pela complementação da bolsa através do programa de bolsa de rendimento acadêmico.

Por fim, a todos aqueles que contribuíram de uma forma ou outra na realização desta Dissertação. 


\section{Resumo}

Orlando, Diego; Gonçalves, Paulo Batista. Absorsor Pendular para

Controle de Vibrações de Torres Esbeltas. Rio de Janeiro, 2006. 168p.

Dissertação de Mestrado - Departamento de Engenharia Civil, Pontifícia Universidade Católica do Rio de Janeiro.

Nesse trabalho, estuda-se o desempenho de um absorsor pendular no controle de vibrações de torres altas e esbeltas, ocasionadas por carregamentos dinâmicos, tais como, por exemplo, cargas ambientais. Em virtude da possibilidade de oscilações de grande amplitude, considera-se na modelagem do problema a não-linearidade do pêndulo. O principal objetivo é estudar o comportamento do sistema torre-pêndulo, submetido a um carregamento harmônico, no regime não-linear, abordando-se aspectos gerais ligados à estabilidade dinâmica. Apresenta-se, inicialmente, a formulação necessária para obter o funcional de energia do sistema coluna-pêndulo, tanto para o caso linear quanto para o caso não-linear, do qual derivam-se as equações diferenciais parciais de movimento. A partir das equações lineares, obtêm-se as freqüências naturais e modos de vibração para alguns casos relevantes de coluna. A seguir, com base na análise modal do sistema coluna-pêndulo, deriva-se um modelo de dois graus de liberdade capaz de descrever com precisão o comportamento do sistema na vizinhança da freqüência fundamental da coluna, do qual obtêm-se as equações de movimento e as equações de estado não-lineares. Uma análise paramétrica detalhada das oscilações não-lineares do sistema coluna-pêndulo demonstra que o absorsor pendular passivo pode reduzir ou amplificar a resposta da coluna. No estudo da influência da não-linearidade geométrica do pêndulo, verifica-se a importância dessa na resposta do sistema, evidenciando que a nãolinearidade não pode ser desprezada nessa classe de problema. Por fim, com base nos resultados, propõe-se um absorsor pendular híbrido. Os estudos revelam que este controle é mais eficiente que o passivo e que não requer grande gasto de energia.

\section{Palavras-chave}

Torres esbeltas, absorsor dinâmico de vibrações, absorsor pendular, controle de vibrações, oscilações não-lineares, estabilidade dinâmica. 


\section{Abstract}

Orlando, Diego; Gonçalves, Paulo Batista. Vibration Control of Slender Towers with a Pendulum Absorber. Rio de Janeiro, 2006. 168p. MSc. Dissertation - Department of Civil Engineering, Pontifícia Universidade Católica do Rio de Janeiro.

In the present work the performance of a pendulum absorber in the vibration control of tall and slender towers, caused by dynamic loads, such as, environmental loads, is studied in detail. Due to the possibility of large amplitude oscillations, the non-linearity of the pendulum is considered in the modeling of the problem. The main objective of this research is to study the behavior of the tower-pendulum system, submitted to a harmonic load, in the nonlinear regimen, with emphasis on general aspects related to its dynamic stability. It is presented, initially, the formulation necessary for the derivation of the system's energy functional, both for the linear and the nonlinear cases, from which the partial differential equations of motion are derived and the vibration frequencies and related vibration modes are obtained. Then, based on the modal analysis of the column-pendulum system, a two degrees of freedom model, capable of describing with precision the behavior of the system in the neighborhood of the fundamental frequency of the column is derived, from which the equations of motion and the nonlinear state-space equations are obtained. A detailed parametric analysis of the nonlinear oscillations of the system is carried out. It shows that the pendulum may reduce or amplify the response of the column. The results show a marked influence of the geometric not-linearity of the pendulum on the response of the system, showing that its not-linearity cannot be neglected in this class of problems. Finally, based on the results, a hybrid control approach is proposed. These studies show that this control strategy is more efficient than the passive control alone and that it does not require a large amount of energy.

\section{Keywords}

Slender towers, dynamic vibration absorber, pendulum absorber, vibration control, nonlinear oscillations, dynamic stability. 


\section{Sumário}

1 Introdução $\quad 27$

1.1. Motivação 32

1.2. Objetivos 33

1.3. Organização do Trabalho 33

2 Formulação do Problema 35

2.1. Funcional de Energia do Sistema - Formulação Não-Linear 36

2.1.1. Energia Potencial Total da Coluna 37

2.1.2. Energia Cinética da Coluna 42

2.1.3. Amortecimento da Coluna 43

2.1.4. Força Harmônica 44

2.1.5. Funcional de Energia da Coluna - Formulação Não-Linear 44

2.1.6. Funcional de Energia do Pêndulo - Formulação Não-Linear 44

2.1.7. Montagem do Funcional de Energia do Sistema - Formulação $\begin{array}{ll}\text { Não-Linear } & 47\end{array}$

2.2. Funcional de Energia do Sistema - Coluna Linear 47

2.3. Dedução das Equações Diferenciais de Movimento 48

3 Freqüências Naturais e Modos de Vibração da Coluna 50

3.1. Coluna de Seção Constante sem Força Axial 50

3.1.1. Estudo das Freqüências Naturais $\quad 57$

3.1.2. Estudo dos Modos de Vibração 60

3.2. Coluna de Seção Variável com Força Axial 62

3.2.1. Avaliação da Força Axial 66

3.2.2. Exemplo Numérico $\quad 67$

3.2.2.1. Coluna sem o Efeito do Peso Próprio 69

3.2.2.2. Coluna com o Efeito do Peso Próprio 69

4 Solução do Sistema Coluna-Pêndulo 71

4.1. Solução Modal 
4.2. Exemplo 72

4.3. Justificativa para o Modelo de dois Graus de Liberdade 76

4.3.1. Equações Não-Lineares do Modelo de Dois Graus de Liberdade 76

4.4. Correlação com o Modelo Discreto de Dois Graus de Liberdade 76

4.5. Relação Freqüência-Amplitude da Coluna com Pêndulo Absorsor

5 Estudo Paramétrico do Sistema Coluna-Pêndulo

5.1. Influência da Freqüência da Excitação no Comportamento do Sistema

5.2. Influência da Freqüência do Pêndulo no Comportamento do Sistema

5.3. Influência das Condições Iniciais do Pêndulo Absorsor no Comportamento do Sistema 100

5.3.1. Resposta do Sistema a um Carregamento Senoidal 100

5.3.2. Comportamento do Sistema sob um Pulso Senoidal 104

5.3.3. Comportamento do Sistema sob um Pulso Retangular 105

5.3.4. Comportamento do Sistema para uma Velocidade Inicial 106

5.4. Influência do Amortecimento do Pêndulo no Comportamento do Sistema 107

5.5. Influência de uma Mola com Rigidez Linear 108

5.5.1. Variação da Rigidez Linear 109

5.5.2. Efeito de uma Mola Não-Linear 111

6 Resposta do Sistema Não-Linear 114

6.1. Obtenção das Equações Algébricas Não-Lineares 114

6.2. Resultados Numéricos 117

6.2.1. Exemplo 1

6.2.2. Exemplo 2

7 Absorsor Dinâmico de Vibrações Híbrido 139 
7.1. Comportamento do Sistema em Função dos Parâmetros da Força de Controle

7.1.1. Influência do parâmetro $f$

$\begin{array}{ll}\text { 7.1.2. Influência do parâmetro } \beta & 147\end{array}$

7.2. Comportamento do Sistema Considerando Defasagem no Cálculo da Força de Controle 151

7.3. Comportamento do Sistema para um Pulso Retangular 155

7.4. Comportamento do Sistema para um Pulso com Amplitude Variável

8 Conclusões e Sugestões 160

8.1. Conclusões 160

8.2. Sugestões 161

9 Referências Bibliográficas 162 


\section{Lista de Figuras}

Figura 1.1: Torres de telecomunicações. 27

Figura 1.2: Desprendimento de vórtices (Techet, 2005). 28

Figura 2.1: Coluna em estudo.

Figura 2.2: Deslocamento transversal e encurtamento da coluna. $\quad 38$

Figura 2.3: Elemento infinitesimal da linha neutra da viga. 38

Figura 2.4: Parâmetros do pêndulo. $\quad 45$

Figura 3.1: Coluna de seção constante sem força axial. 51

Figura 3.2: Modos de vibração da coluna. 54

Figura 3.3: Parcelas da condição de continuidade do esforço

cortante. $\quad 55$

Figura 3.4: Variação da primeira freqüência em função de $\alpha$ e $v . \quad 58$

Figura 3.5: Variação da segunda freqüência em função de $\alpha$ e $v$. $\quad 59$

Figura 3.6: Variação da terceira freqüência em função de $\alpha$ e $v$. $\quad 59$

Figura 3.7: Comparação entre as três primeiras freqüências

quando $v=1$.

Figura 3.8: Forma do primeiro modo de vibração variando-se $v$. 61

Figura 3.9: Forma do segundo modo de vibração variando-se $v$. 61

Figura 3.10: Forma do terceiro modo de vibração variando-se $v$. 62

Figura 3.11: Coluna de seção variável com força axial. 63

Figura 3.12: Variação da força axial (Li et al., 2000). 67

Figura 3.13: Coluna do exemplo numérico. 68

Figura 3.14: Modos de vibração da coluna sem o efeito do peso próprio.

Figura 3.15: Modos de vibração da coluna com o efeito do peso próprio. $\quad 70$

Figura 4.1: Exemplo em estudo. 72

Figura 4.2: Modos de vibração do sistema coluna-pêndulo. 75

Figura 4.3: Sistema discreto massa-pêndulo. 77 
Figura 4.4: Comportamento do fator de amplificação de deslocamento da coluna.

Figura 4.5: Comportamento do fator de amplificação da rotação no topo da coluna.

Figura 4.6: Comportamento do fator de amplificação de deslocamento da coluna para o ajuste ótimo.

Figura 4.7: Comportamento do fator de amplificação de deslocamento da coluna para diferentes relações de $\mu$.

Figura 5.1: Espectro de resposta de deslocamento do sistema para $\omega_{p} / \omega_{c}=0.7965$.

Figura 5.2: Espectro de resposta de deslocamento do sistema para $\omega_{p} / \omega_{c}=1.00$.

Figura 5.3: Espectro de resposta de deslocamento do sistema para $\omega_{p} / \omega_{c}=1.1151$.

Figura 5.4: Espectro de resposta de deslocamento da coluna para $\omega_{p} / \omega_{c}=0.7965$.

Figura 5.5: Espectro de resposta de deslocamento da coluna para $\omega_{p} / \omega_{c}=1.00$.

Figura 5.6: Espectro de resposta de deslocamento da coluna para $\omega_{p} / \omega_{c}=1.1151$.

Figura 5.7: Variação das amplitudes máximas de deslocamento da coluna original e com absorsor na resposta permanente.

Figura 5.8: Diagramas de bifurcação para o deslocamento da coluna na resposta permanente.

Figura 5.9: Resposta no tempo, plano fase e seção de Poincaré da resposta permanente da coluna.

Figura 5.10: Diagramas de bifurcação para o deslocamento angular do pêndulo na resposta permanente.

Figura 5.11: Resposta no tempo, plano fase e seção de Poincaré da resposta permanente do pêndulo.

Figura 5.12: Amplitudes máximas da resposta total e permanente da coluna e do pêndulo. 
Figura 5.13: Comportamento das amplitudes durante a resposta permanente.

Figura 5.14: Comportamento da força adimensional F.

Figura 5.15: Comportamento das amplitudes máximas da coluna na resposta total para um carregamento harmônico senoidal.

Figura 5.16: Comportamento das amplitudes máximas da coluna na resposta permanente para um carregamento harmônico senoidal. 101 Figura 5.17: Resposta da coluna no tempo para um carregamento harmônico senoidal.

Figura 5.18: Comportamento das amplitudes máximas do pêndulo na resposta total para um carregamento harmônico senoidal.

Figura 5.19: Comportamento das amplitudes máximas do pêndulo na resposta permanente para um carregamento harmônico senoidal.

Figura 5.20: Resposta do pêndulo no tempo para um carregamento harmônico senoidal.

Figura 5.21: Pulso senoidal.

Figura 5.22: Comportamento das amplitudes máximas da coluna para um pulso senoidal.

Figura 5.23: Comportamento das amplitudes máximas do pêndulo para um pulso senoidal.

Figura 5.24: Pulso retangular.

Figura 5.25: Comportamento das amplitudes máximas da coluna para um pulso retangular.

Figura 5.26: Comportamento das amplitudes máximas da coluna para uma velocidade inicial.

Figura 5.27: Amplitudes de deslocamento da coluna na resposta transiente para diferentes valores de $\xi_{p}$.

Figura 5.28: Influência da variação da taxa de amortecimento do pêndulo nas amplitudes máximas de resposta da coluna e do pêndulo.

Figura 5.29: Comportamento das amplitudes máximas do sistema na resposta total em função da variação de rigidez do pêndulo. 
Figura 5.30: Comportamento das amplitudes máximas do sistema na resposta permanente em função da variação de rigidez do pêndulo.

Figura 6.1: Variação de $\bar{\theta}$ para $\omega_{p} / \omega_{c}=1.0, \xi_{p}=0.0 \%, \mu=0.20 \mathrm{e}$ $F=0.092$.

Figura 6.2: Variação de $\zeta$ para $\omega_{p} / \omega_{c}=1.0, \xi_{p}=0.0 \%, \mu=0.20 \mathrm{e}$ $F=0.092$.

Figura 6.3: Variação do ângulo de fase $\varphi$ para $\omega_{p} / \omega_{c}=1.0$,

$\xi_{p}=0.0 \%, \mu=0.20$ e $F=0.092$.

Figura 6.4: Variação do ângulo de fase $\psi$ para $\omega_{p} / \omega_{c}=1.0$, $\xi_{p}=0.0 \%, \mu=0.20$ e $F=0.092$.

Figura 6.5: Influência do amortecimento do pêndulo em $\bar{\theta}$ e $\zeta$ para $\omega_{p} / \omega_{c}=1.0, \mu=0.20$ e $F=0.092$.

Figura 6.6: Variação de $\bar{\theta}$ para $\omega_{p} / \omega_{c}=0.833, \xi_{p}=26.23 \%$, $\mu=0.20$ e $F=0.041$.

Figura 6.7: Variação do deslocamento angular $\bar{\theta}$ ao longo do tempo para $\omega_{p} / \omega_{c}=0.833, \xi_{p}=26.23 \%, \mu=0.20$ e $F=0.041$.

Figura 6.8: Variação de $\zeta$ para $\omega_{p} / \omega_{c}=0.833, \xi_{p}=26.23 \%$, $\mu=0.20$ e $F=0.041$.

Figura 6.9: Variação do deslocamento $\zeta$ ao longo do tempo para $\omega_{p} / \omega_{c}=0.833, \xi_{p}=26.23 \%, \mu=0.20$ e $F=0.041$.

Figura 6.10: Variação do ângulo de fase $\varphi$ para $\omega_{p} / \omega_{c}=1.0$, $\xi_{p}=26.23 \%, \mu=0.20$ e $F=0.041$.

Figura 6.11: Variação do ângulo de fase $\psi$ para $\omega_{p} / \omega_{c}=1.0$, $\xi_{p}=26.23 \%, \mu=0.20$ e $F=0.041$.

Figura 6.12: Variação das amplitudes de deslocamento $\bar{\theta}$ e $\zeta\left(\bar{x} / x_{\text {est }}\right)$ para $\omega_{p} / \omega_{c}=0.833, \xi_{p}=26.23 \%, \mu=0.20 \mathrm{e}$ $F=0.041$ (Pinheiro, 1997). 
Figura 6.13: Influência da não-linearidade do pêndulo em $\bar{\theta}$ e $\zeta$ para $\omega_{p} / \omega_{c}=1.0, \mu=0.20$ e $F=0.092$.

Figura 6.14: Influência da não-linearidade do pêndulo em $\bar{\theta}$ e $\zeta$ para $\omega_{p} / \omega_{c}=1.0, \mu=0.20, F=0.092$ e $\xi_{\text {pótimo }}=0.25$.

Figura 6.15: Amplitudes de deslocamento angular $\bar{\theta}$ para $\omega_{p} / \omega_{c}=1.018, \xi_{p}=0.0 \%, \mu=0.04$ e $\zeta_{s}=0.007$.

Figura 6.16: Diagrama de bifurcação do mapa de Poincaré.

Variação da coordenada $\bar{\theta}$ para $\omega_{p} / \omega_{c}=1.018, \xi_{p}=0.0 \%$, $\mu=0.04$ e $\zeta_{s}=0.007$.

Figura 6.17: Amplitudes de deslocamento $\zeta$ para $\omega_{p} / \omega_{c}=1.018$, $\xi_{p}=0.0 \%, \mu=0.04$ e $\zeta_{s}=0.007$.

Figura 6.18: Diagrama de bifurcação do mapa de Poincaré.

Variação da coordenada $\zeta$ para $\omega_{p} / \omega_{c}=1.018, \xi_{p}=0.0 \%$, $\mu=0.04$ e $\zeta_{s}=0.007$.

Figura 6.19: Amplitudes de deslocamento angular $\bar{\theta}$ para $\omega_{p} / \omega_{c}=1.018, \xi_{p}=7.0 \%, \mu=0.04$ e $\zeta_{s}=0.007$.

Figura 6.20: Diagrama de bifurcação do mapa de Poincaré.

Variação da coordenada $\bar{\theta}$ para $\omega_{p} / \omega_{c}=1.018, \xi_{p}=7.0 \%$, $\mu=0.04$ e $\zeta_{s}=0.007$.

Figura 6.21: Variação do deslocamento angular $\bar{\theta}$ ao longo do tempo para $\omega_{p} / \omega_{c}=1.018, \xi_{p}=7.0 \%, \mu=0.04$ e $\zeta_{s}=0.007$.

Figura 6.22: Amplitude de deslocamento $\zeta$ para $\omega_{p} / \omega_{c}=1.018$, $\xi_{p}=7.0 \%, \mu=0.04$ e $\zeta_{s}=0.007$.

Figura 6.23: Diagrama de bifurcação do mapa de Poincaré.

Variação da coordenada $\zeta$ para $\omega_{p} / \omega_{c}=1.018, \xi_{p}=7.0 \%$, $\mu=0.04$ e $\zeta_{s}=0.007$.

Figura 6.24: Variação do deslocamento $\zeta$ ao longo do tempo para $\omega_{p} / \omega_{c}=1.018, \xi_{p}=7.0 \%, \mu=0.04$ e $\zeta_{s}=0.007$. 
Figura 6.25: Comportamento das amplitudes de deslocamento angular do pêndulo para diferentes valores de $\zeta_{s}$ e $\omega_{p} / \omega_{c}=1.018$, $\xi_{p}=0.0 \%$ e $\mu=0.04$.

Figura 6.26: Comportamento das amplitudes de deslocamento da coluna para diferentes valores de $\zeta_{s}$ e $\omega_{p} / \omega_{c}=1.018$, $\xi_{p}=0.0 \%$ e $\mu=0.04$.

Figura 6.27: Comportamento das amplitudes de deslocamento da coluna original para diferentes valores de $\zeta_{s}$.

Figura 6.28: Comportamento das amplitudes de deslocamento angular do pêndulo para diversos valores de $\zeta_{s}$ e $\omega_{p} / \omega_{c}=1.018$, $\xi_{p}=7.0 \%$ e $\mu=0.04$.

Figura 6.29: Comportamento das amplitudes de deslocamento da coluna para diversos valores de $\zeta_{s}$ e $\omega_{p} / \omega_{c}=1.018, \xi_{p}=7.0 \%$ e $\mu=0.04$.

Figura 7.1: Comportamento da função $\tanh (\beta x)$.

Figura 7.2: Comportamento das amplitudes do sistema e da força de controle.

Figura 7.3: Comparação das amplitudes de deslocamento da coluna, sem e com a força de controle.

Figura 7.4: Comparação das amplitudes de deslocamento angular do pêndulo, sem e com a força de controle.

Figura 7.5: Comportamento das amplitudes de deslocamento da coluna no tempo variando $f$.

Figura 7.6: Comportamento das amplitudes de deslocamento angular do absorsor pendular no tempo variando $f$.

Figura 7.7: Comportamento das amplitudes de deslocamento da coluna no tempo variando $\beta$.

Figura 7.8: Comportamento das amplitudes de deslocamento angular do absorsor pendular no tempo variando $\beta$.

Figura 7.9: Comportamento da função $\operatorname{sign}(x)$. 
Figura 7.10: Variação da amplitude máxima da coluna em função de $\beta$.

Figura 7.11: Variação da amplitude máxima da coluna em função de $f$.

Figura 7.12: Comportamento das amplitudes do sistema com a força de controle para um pulso retangular.

Figura 7.13: Força de excitação da equação (7.3). 


\section{Lista de Tabelas}

Tabela 3.1: Comparação dos resultados.

Tabela 3.2: Freqüências naturais da coluna sem o efeito do peso próprio (rad/s).

Tabela 3.3: Freqüências naturais da coluna com o efeito do peso próprio (rad/s).

70

Tabela 4.1: Freqüências naturais do sistema (rad/s). 74

Tabela 4.2: Modos de vibração do sistema.

Tabela 5.1: Valores máximos da resposta não controlada. 98

Tabela 5.2: Amplitudes de deslocamento da coluna na resposta transiente para diferentes $\xi_{p}$.

107

Tabela 5.3: Variação da relação de freqüências com a variação da rigidez do pêndulo.

109

Tabela 5.4: Amplitudes máximas da coluna na resposta total com a variação de rigidez não-linear.

Tabela 5.5: Amplitudes máximas do pêndulo na resposta total com a variação de rigidez não-linear.

Tabela 5.6: Amplitudes máximas da resposta da coluna na fase permanente em função da variação de rigidez não-linear Tabela 5.7: Amplitudes máximas da resposta do pêndulo na fase permanente em função da variação de rigidez não-linear.

Tabela 6.1: Comparação das amplitudes máximas obtidas no domínio da freqüência e no domínio do tempo para $\omega_{p} / \omega_{c}=0.833, \xi_{p}=26.23 \%, \mu=0.20$ e $F=0.041$.

Tabela 6.2: Comparação das amplitudes máximas obtidas no domínio da freqüência e no domínio do tempo para $\omega_{p} / \omega_{c}=1.018, \xi_{p}=7.0 \%, \mu=0.04$ e $\zeta_{s}=0.007$.

Tabela 7.1: Influência do parâmetro $f$ nas amplitudes máximas da coluna na resposta total. 
Tabela 7.2: Influência do parâmetro $f$ nas amplitudes máximas da coluna na resposta permanente.

Tabela 7.3: Influência do parâmetro $f$ nas amplitudes máximas do pêndulo na resposta total.

Tabela 7.4: Influência do parâmetro $f$ nas amplitudes máximas do pêndulo na resposta permanente.

Tabela 7.5: Influência do parâmetro $\beta$ nas amplitudes máximas da coluna na resposta total.

Tabela 7.6: Influência do parâmetro $\beta$ nas amplitudes máximas da coluna na resposta permanente.

Tabela 7.7: Influência do parâmetro $\beta$ nas amplitudes máximas do pêndulo na resposta total.

Tabela 7.8: Influência do parâmetro $\beta$ nas amplitudes máximas do pêndulo na resposta permanente.

Tabela 7.9: Influência da defasagem nas amplitudes máximas da coluna na resposta total para $f=1.00$ e $\beta=6000$.

Tabela 7.10: Influência da defasagem nas amplitudes máximas da coluna na resposta permanente para $f=1.00$ e $\beta=6000$.

Tabela 7.11: Influência da defasagem nas amplitudes máximas do pêndulo na resposta total para $f=1.00$ e $\beta=6000$.

Tabela 7.12: Influência da defasagem nas amplitudes máximas do pêndulo na resposta permanente para $f=1.00$ e $\beta=6000$.

Tabela 7.13: Influência da defasagem nas amplitudes máximas da coluna na resposta total para $f=1.00$ e $\beta=60$.

Tabela 7.14: Influência da defasagem nas amplitudes máximas da coluna na resposta permanente para $f=1.00$ e $\beta=60$.

Tabela 7.15: Influência da defasagem nas amplitudes máximas do pêndulo na resposta total para $f=1.00$ e $\beta=60$.

Tabela 7.16: Influência da defasagem nas amplitudes máximas do pêndulo na resposta permanente para $f=1.00$ e $\beta=60$.

Tabela 7.17: Influência da duração do pulso retangular na resposta da coluna. 
Tabela 7.18: Influência da duração do pulso retangular na resposta do pêndulo.

Tabela 7.19: Influência do parâmetro $\varepsilon_{0}$ nas amplitudes máximas da coluna.

Tabela 7.20: Influência do parâmetro $\varepsilon_{0}$ nas amplitudes máximas do pêndulo. 


\section{Lista de Símbolos}

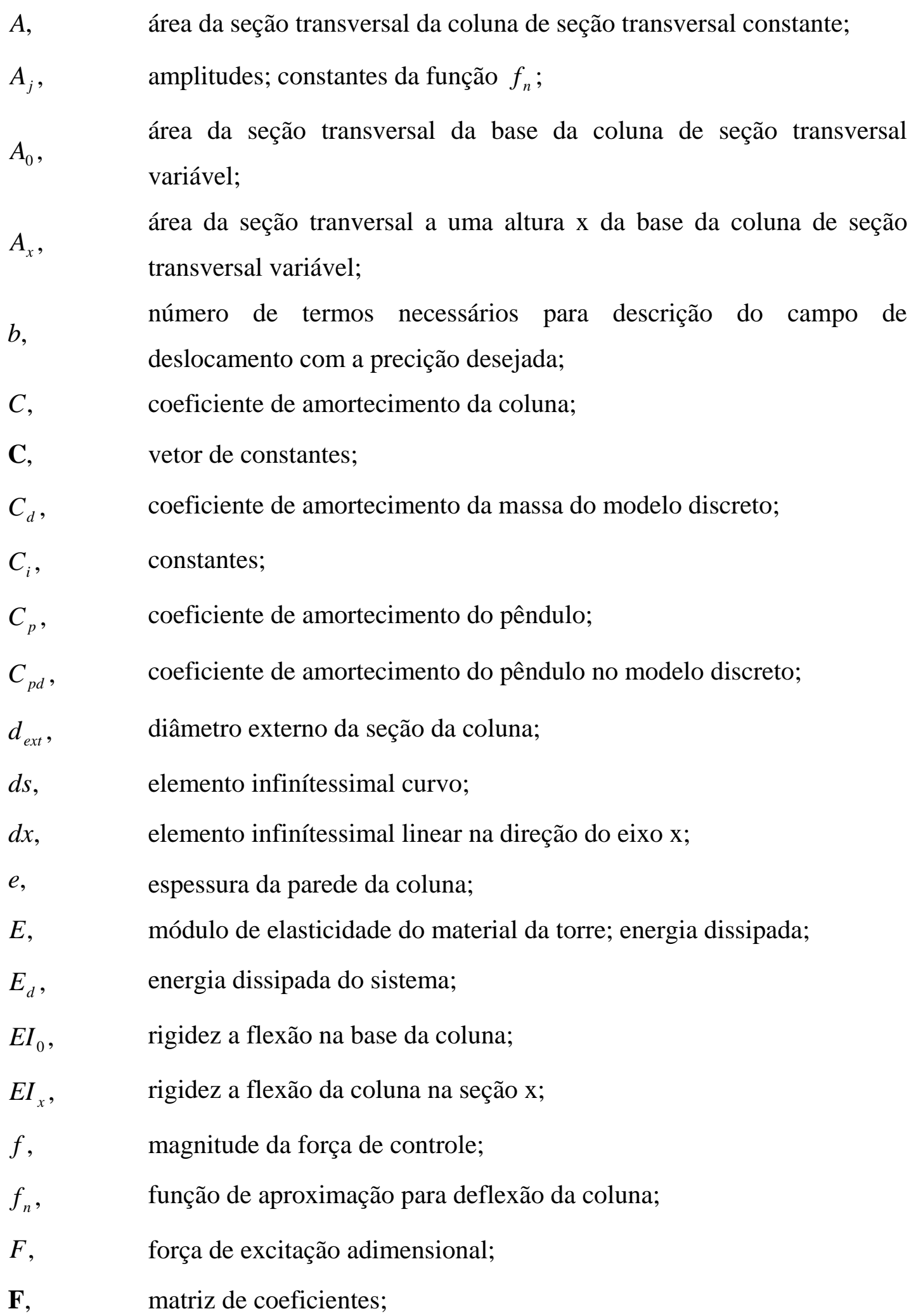


$F A_{\zeta}$, fator de amplificação de deslocamento da coluna;

$F A_{\psi}$, fator de amplificação de rotação da coluna;

$F A_{\zeta_{-} o ́ t i m o}, \quad$ fator de amplificação da coluna ótimo;

Fc, força de controle;

$F_{e}$, força de excitação para uma explosão, ou terremoto, ou rajada de vento;

$F_{o}, \quad$ amplitude da força de excitação;

g, aceleração da gravidade;

I, momento de inércia da seção transversal da coluna;

$I_{n}$, $\quad$ função de Bessel de terceiro tipo;

$I_{x}$, momento de inércia da seção transversal da coluna, na seção x;

l, comprimento da haste do pêndulo absorsor;

$l_{d}$, comprimento da haste do pêndulo no modelo discreto;

$J_{n}, J_{i}, \quad$ função de Bessel de primeiro tipo;

L, $\quad$ comprimento da coluna;

$L_{1}$, comprimento da extremidade engastada até a massa concentrada $M_{c}$;

$L_{2}$, comprimento da massa concentrada $M_{c}$ até a extremidade livre da coluna;

K, matriz de rigidez do sistema coluna-pêndulo;

$K_{d}$, $\quad$ rigidez elástica da massa do modelo discreto;

$K_{n}$, função de Bessel de quarto tipo;

$K_{n l}$, $\quad$ rigidez não-linear do pêndulo absorsor;

$K_{p}$, $\quad$ rigidez torsional do pêndulo absorsor;

$K_{p d}$, rigidez torsional do pêndulo do modelo discreto;

m, massa do pêndulo absorsor;

$m_{d}$, massa do pêndulo do modelo discreto;

M, massa por unidade de comprimento na coluna da seção transversal constante;

M, matriz de massa do sistema coluna-pêndulo; 


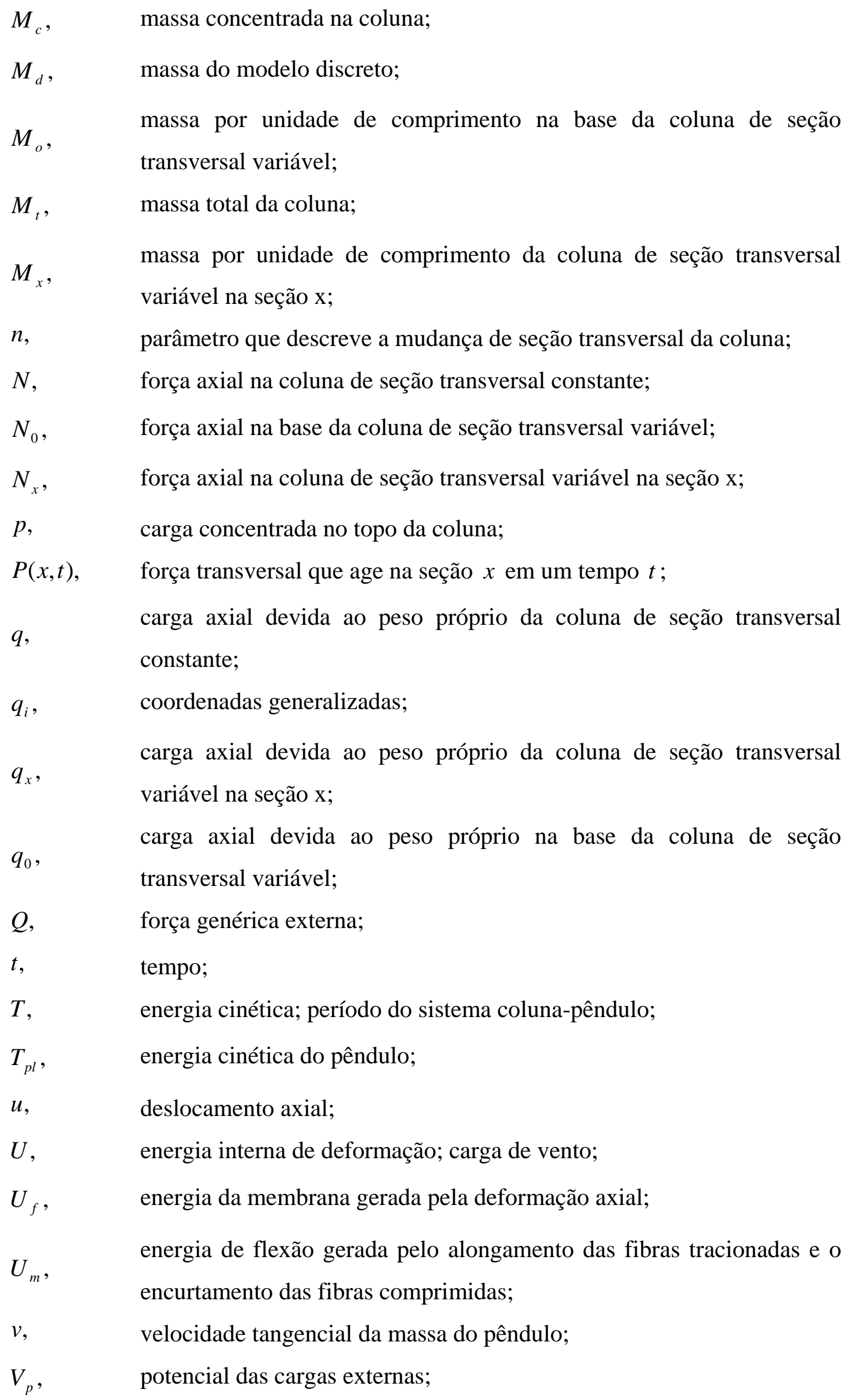




\begin{tabular}{|c|c|}
\hline$V_{p l}$, & energia potencial total do pêndulo; \\
\hline$Y_{n}(d)$ & função de Bessel de segundo tipo; \\
\hline$x$, & coordenada axial; \\
\hline$w$, & deslocamento transversal da coluna; \\
\hline $\bar{w}$ & deslocamento transversal da coluna; \\
\hline$w_{e s}$, & deslocamento estático da coluna; \\
\hline$W$, & trabalho; \\
\hline$W_{n c}$ & trabalho realizado pelas forças não conservativas; \\
\hline$W_{p}$ & trabalho realizado pela força harmônica; \\
\hline$\frac{1}{R_{0}}$ & curvatura da estrutura indeformada; \\
\hline$\frac{1}{R_{f}}$ & curvatura do eixo deformado; \\
\hline$\alpha$ & $\begin{array}{l}\text { relação entre a massa concentrada }\left(M_{c}\right) \text { e massa total da coluna }\left(M_{t}\right) \text {; } \\
\text { parâmetro de controle da rigidez não-linear do pêndulo; }\end{array}$ \\
\hline$\beta$ & parâmetro de controle da força de controle; \\
\hline$\beta_{j}$ & parâmetro de freqüência; \\
\hline$\delta$, & variação dos termos; função delta de Dirac; \\
\hline$\varepsilon$ & deformação específica da linha neutra; \\
\hline$\varepsilon_{0}$ & parâmetro de controle da força de excitação $F_{e}$; \\
\hline$\zeta$ & parâmetro adimensional de deslocamento da coluna; \\
\hline$\zeta_{s}$ & $\begin{array}{l}\text { parâmetro adimensional de deslocamento estático; amplitude da força } \\
\text { de excitação (adimensional); }\end{array}$ \\
\hline$\zeta_{\text {orig }}$ & parâmetro adimensional de deslocamento da coluna original; \\
\hline$\eta$ & $\begin{array}{l}\text { parâmetro que descreve a mudança de seção transversal da coluna; } \\
\text { relação entre a freqüência de excitação e a freqüência natural da } \\
\text { coluna; }\end{array}$ \\
\hline$\theta$ & deslocamento angular do pêndulo absorsor; \\
\hline $\bar{\theta}$ & deslocamento angular do pêndulo absorsor; \\
\hline$\theta_{0}$, & condição inicial do deslocamento angular do pêndulo absorsor; \\
\hline
\end{tabular}




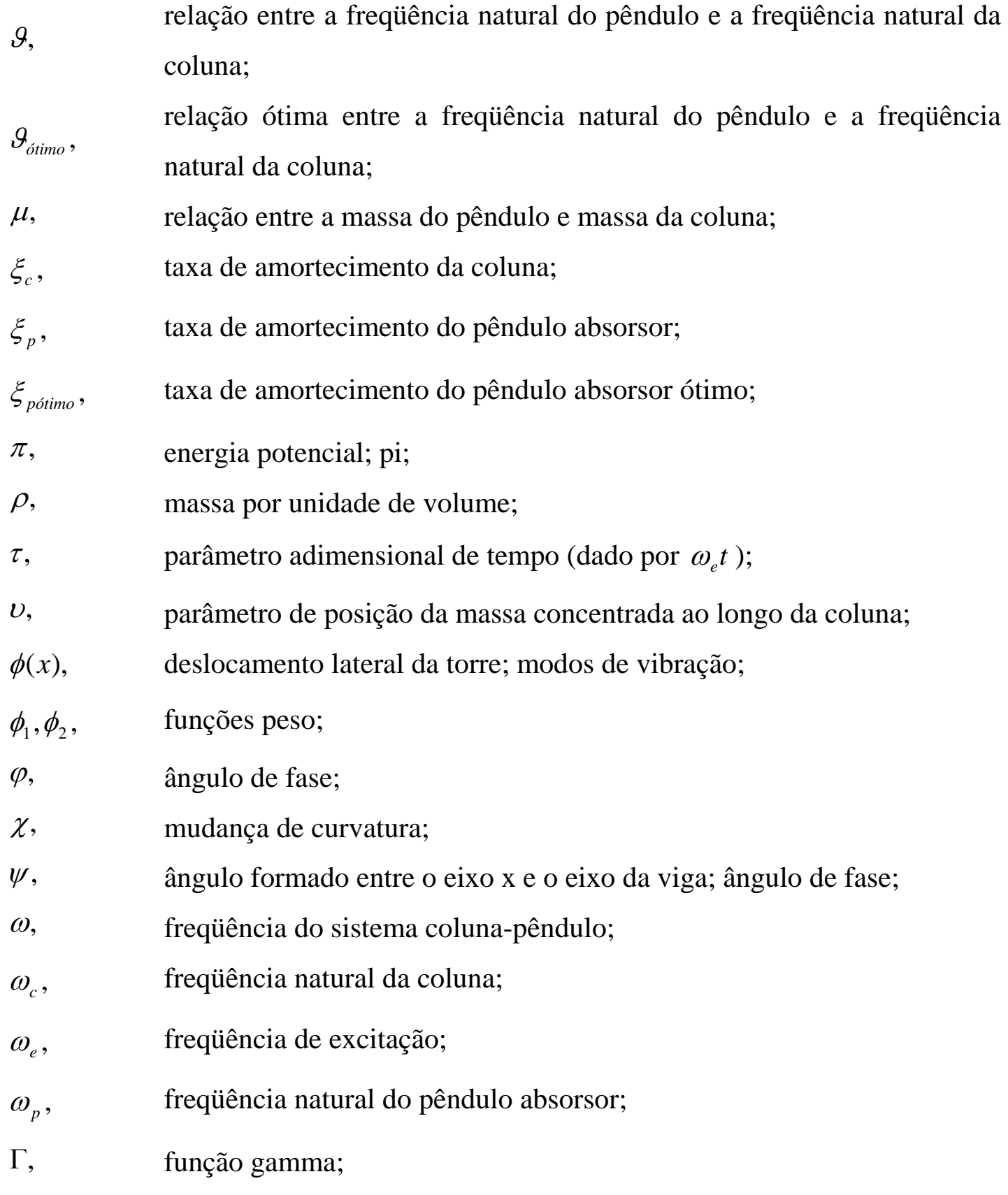

$\Delta, \quad$ encurtamento na extremidade da coluna. 